\title{
Mobile Multi-Actuator Tactile Displays
}

\author{
Eve Hoggan, Sohail Anwar and Stephen A. Brewster \\ Glasgow Interactive Systems Group, Department of Computing Science \\ University of Glasgow, Glasgow, G12 8QQ, UK \\ \{eve, stephen\}@dcs.gla.ac.uk www.tactons.org
}

\begin{abstract}
The potential of using the sense of touch to communicate information in mobile devices is receiving more attention because of the limitations of graphical displays in such situations. However, most applications only use a single actuator to present vibrotactile information. In an effort to create richer tactile feedback and mobile applications that make use of the entire hand and multiple fingers as opposed to a single fingertip, this paper presents the results of two experiments investigating the perception and application of multiactuator tactile displays situated on a mobile device. The results of these experiments show that an identification rate of over $87 \%$ can be achieved when two dimensions of information are encoded in Tactons using rhythm and location. They also show that location produces $100 \%$ recognition rates when using actuators situated on the mobile device at the lower thumb, upper thumb, index finger and ring finger. This work demonstrates that it is possible to communicate information through four locations using multiple actuators situated on a mobile device when non-visual information is required.
\end{abstract}

Keywords: Multimodal Interaction, Haptic I/O, Tactile Icons (Tactons), Mobile Displays, Multi-Actuator Displays.

\section{Introduction}

Tactile displays for mobile devices are becoming an important area of research in multimodal interaction. Mobile devices like PDAs and smart phones are becoming ever more popular as they provide many of the conveniences of a desktop computer in a mobile setting. However, they can be much more difficult to use due to demands on visual attention and limited screen space. Although audio output has proven to be an effective feedback modality for mobile devices, it can sometimes be inappropriate or go unnoticed, especially in a noisy environment like a concert or train station, or in a situation where it would be socially improper for a mobile device to ring loudly e.g. in a library or business meeting. These devices already commonly incorporate a vibrotactile actuator but with only very simple feedback. The potential of using the sense of touch to communicate information from mobile devices has already generated a body of research exploring various techniques such as vibrotactile icons (Tactons), lateral skin stretch display platforms, wearable displays and haptic pens [4, 9, 10, 12]. This research has shown that the sense of touch is a powerful communication medium for mobile devices and that users can understand information encoded in the tactile modality. However, these applications tend to involve only a single actuator to present 
vibrotactile information. Given the promising results from existing research, it may be possible to increase the bandwidth of information by investigating the use of multiple actuators to present information.

Typically, humans are accustomed to performing exploratory or manipulation tasks by using their hands and fingers [8]. The sensitivity of the human hand is based on a multitude of receptors embedded inside the fingertips and we gain a rich understanding of the objects we hold and touch. State-of-the-art tactile feedback displays for mobile device users already ensure high feedback quality, but often only for a singlefingered display or for a display with multiple actuators positioned on the body.

In an effort to create tactile feedback and mobile applications that make use of the entire hand and multiple fingers as opposed to a single fingertip, this paper presents the results from two experiments investigating the perception and application of multi-actuator tactile displays situated on a mobile device.

In related work, with regard to the feedback provided by the multi-actuator display, the output was based on previous research findings in Tactons work [3]. Tactons are structured vibrotactile messages which can be used to communicate information nonvisually. They are the tactile equivalent of audio and visual icons. Tactons have been designed to be used in situations where the display may be overloaded, limited or unavailable, and when audio cues are not appropriate. Research carried out by Brown [4] showed that Tactons are effective as a means of communication and that by combining parameters like rhythm, spatial location and roughness, several dimensions of information can be presented to the sense of touch. The spatial locations used in Tactons research were on the forearm of mobile device users. This research will develop the spatial location parameter further by using positions on the actual device as opposed to positions on the user.

\section{The Multi-Actuator Mobile Device}

We attached 4 EAI C2 Tactors (www.eaiinfo.com) to a standard PDA to provide vibrotactile feedback. Due to the number of $\mathrm{C} 2$ actuators required on the PDA to carry out the experiment, it was not possible to simply attach them to a PDA as this proved to be uncomfortable for the participants. Instead, the PDA was encased in a protective transparent cover and the actuators were embedded in the cover resulting in a more comfortable but still realistic experience (Figure 1). Only the central part of the $\mathrm{C} 2$ vibrates, meaning that any vibration is localised and does not spread out across the whole device.

The $\mathrm{C} 2$ actuators were placed in four different positions on the PDA corresponding to locations on the hand: the lower thumb (bottom left side), the upper thumb (top left side), the tip of the index finger (top right on the back of the PDA) and the tip of the ring finger (middle right side). These locations were identified by observing how people held the PDA and where their hands made best contact with it. The actuators were controlled via a desktop PC using an M-Audio multi-out soundcard. 

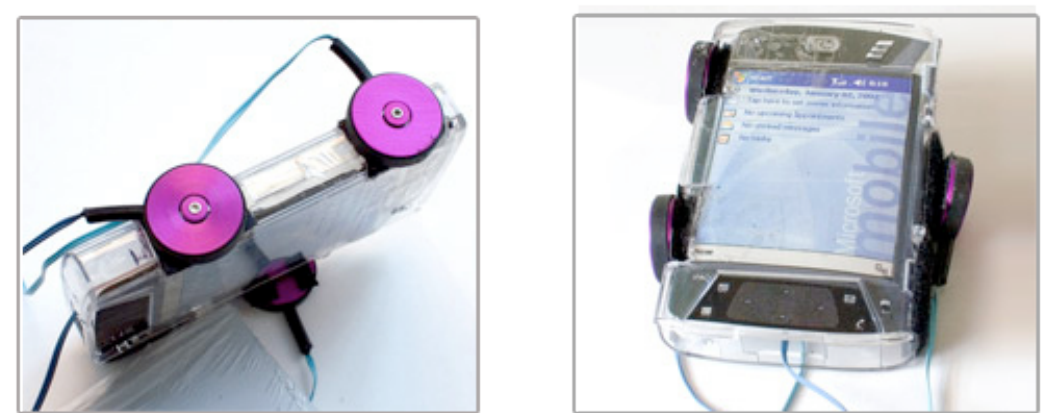

Figure 1. The multi-actuator PDA used in experiment.

\section{Experiment 1 - Potential Locations on the Hand}

The first experiment was conducted to evaluate the absolute identification of Tactons using two parameters: spatial location and rhythm. The aim of this experiment was to investigate the potential of using multiple locations on the same hand for tactile feedback from a mobile device, therefore providing data on how many and which of the locations would be effective. Headphones were worn by the participants to block out any residual sound from the device, to ensure that the participants were responding only to the tactile sensations and not to any audio leakage.

The set of Tactons used in this experiment consisted of three different rhythms (Figure 2) with each of the four spatial locations. There were therefore 12 different Tactons presented 3 times to give 36 tasks in the experiment. Each lasted approximately 1 second and the rhythms used were based on those from Brown et al. [4].

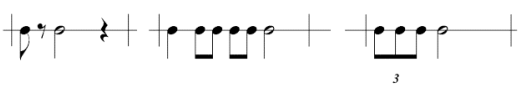

Figure 2. Two note, four note and six note rhythms used in the experiment (from [4]).

Fifteen participants, all of whom were students at the University, took part in this experiment. Before beginning, all were given a tutorial to introduce the concepts of Tactons, rhythm, location, etc. Participants held the PDA in their non-dominant hand. In each task participants were presented with one Tacton and had to identify both attributes (the rhythm and the spatial location) encoded in it. They indicated their response by clicking on the corresponding radio buttons shown in Figure 3. Once they had made their response they clicked the "Submit" button. 


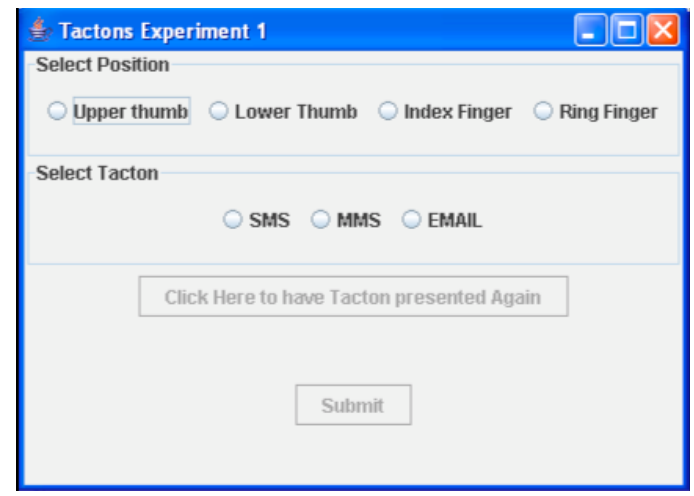

Figure 3. Experiment location and rhythm selection experiment screenshot.

\subsection{Results}

During the experiment data were collected on the number of correct identifications of rhythm and location. Percentage correct scores were calculated for each individual dimension (rhythm and spatial location) and for the complete Tactons. To correctly identify a complete Tacton, both of the individual dimensions had to be correctly identified. The average recognition rate for each location is shown in Figure 4.

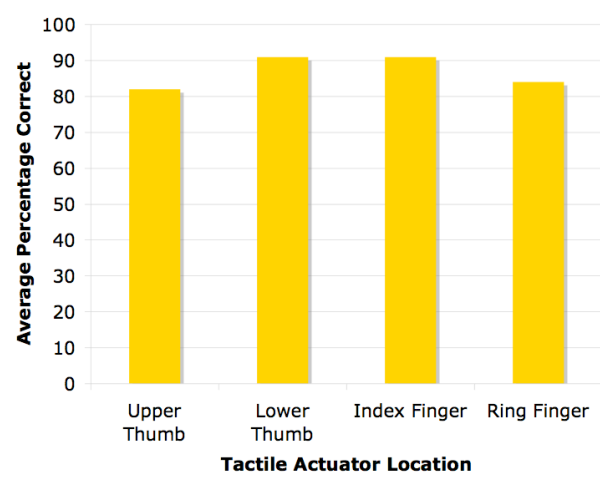

Figure 4. Average percentage correct scores for each tactile actuator location.

The average recognition rate for Tactons on the upper thumb was $82 \%$, the ring finger $84 \%$ and $91 \%$ for both the lower thumb and index finger. Analysis of the data for each of the individual parameters (rhythm and location) showed that the average recognition rates for each location were $100 \%$. No participant had a problem identifying the location of a Tacton. This result suggests that location on the hand is a very effective cue for a tactile display. The rhythm parameter was more difficult to identify and therefore reduced the overall Tacton recognition rates. 


\subsection{A Closer Look at Rhythm}

The results of overall Tacton recognition showed an average recognition rate of $87.2 \%$, with the 2-note rhythm having the highest recognition rate at $98.3 \%$ and the 6-note rhythm having the lowest at $81.6 \%$. Table 1 shows the results for each rhythm, in the form of a confusion matrix which shows the number of times one stimulus was confused for another (as the location parameter got 100\% recognition, no location was ever confused with another).

\begin{tabular}{|l|l|l|l|}
\hline & \multicolumn{1}{|c|}{ 2-Note Rhythm } & 4-Note Rhythm & 6-Note Rhythm \\
\hline 2-Note Rhythm & $98.3 \%$ & $0.9 \%$ & $0.8 \%$ \\
\hline 4-Note Rhythm & $3.3 \%$ & $83.3 \%$ & $13.3 \%$ \\
\hline 6-Note Rhythm & $0 \%$ & $18.3 \%$ & $81.6 \%$ \\
\hline
\end{tabular}

Table 1. Stimulus-response confusion matrix for rhythm.

This shows that the participants experienced confusion attempting to distinguish between the 6-note Tacton and the 4-note one. There was rarely confusion between the 2-note Tacton and any other with the average recognition rate of $98.33 \%$ for the 2note. The differences between each rhythm were investigated using a standard twotailed one-factor ANOVA analysis. The results of the ANOVA indicate that there were no significant differences in error rates between three different rhythms with $\mathrm{F}$ $=4.06$ where $\mathrm{p}=0.05$.

The results of this experiment indicate that spatial location using multiple actuators situated on the mobile device held in the non-dominant hand is an extremely effective parameter when transferring information to the hand (with identification rates of $100 \%$ ). Therefore it can be concluded that these locations are able to successfully detect tactile information and these results are encouraging for further research in the transfer of information to the hand. The results for the individual locations are perfect and although the results for rhythm identification are lower, they are still comparable with results in previous research focusing on single actuator displays where Brown [5] found recognition rates of $93 \%$ for similar rhythms.

\section{Experiment 2 - A More Realistic Application}

Given the good results obtained in the first experiment, a second experiment was carried out involving multi-actuator tactile feedback in a more realistic application. As the multi-actuator set up allows more communication space, sensory saltation [7] could be used. The experiment conducted investigated the effectiveness of a tactile progress bar with error notification using a multi-actuator display.

\subsection{Sensory Saltation}

Sensory Saltation [7], also known as the 'Cutaneous Rabbit', usually involves three actuators placed at equal distances on the forearm, three brief pulses are delivered to the first actuator, followed by three more at the middle, followed by a final three at 
the last actuator. Instead of experiencing the successive stimulations as isolated events, the subject commonly feels that the pulses seem to be distributed with uniform spacing from the site of the first actuator to that of the third (Figure 5). An important and useful feature of sensory saltation is the ability to simulate higher spatial resolution than the actual spacing of actuators. This has already been used successfully in wearable computing for applications such as directional cueing [12]. In this experiment, instead of using the forearm, the actuators are placed on the device held in the hand.

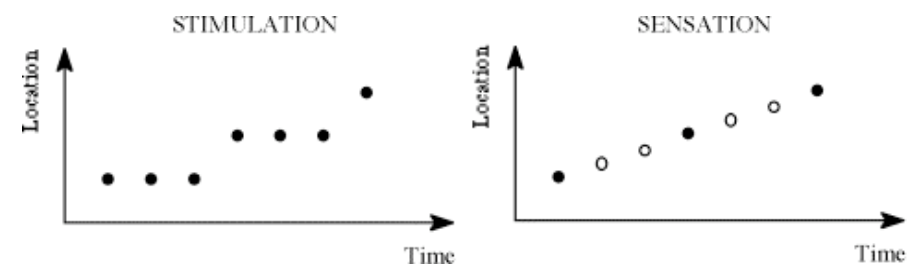

Figure 5. Graphical representation of sensory saltation illusion [7].

\subsection{The Application}

Progress bars are common and widely used to indicate the current state of a task which does not complete instantaneously, such as loading a Web page or copying files. However, there are a number of problems associated with such information when presented visually, for example, visual progress bars may become hidden behind other windows on the desktop and they must compete for screen space and visual attention with other visual tasks such as document editing or Web browsing. This problem is amplified on a mobile device such as a PDA or mobile phone due to the extremely limited screen space. In addition, the user may also need to concentrate on other tasks such as walking or navigating the environment whilst a potentially time consuming task takes place. Myers [11] showed that people prefer systems with progress indicators, as they give novices confidence that a task is progressing successfully whilst experts can get sufficient information to predict the approximate completion time of the task.

Research has already been conducted into the design of audio progress indicators, which give the user information about progress using non-speech sound, avoiding the problems of limited screen space. For example, Gaver [6] used the sound of liquid pouring from one container to another to indicate copying. However, such approaches have some drawbacks; for example, in a noisy environment cues may be missed, equally in a quiet environment the user may not wish to disturb others nearby. Previously research by Brewster and King [1] used Tactons to present progress information in desktop applications using a single actuator. They encoded the amount of download remaining in the time between two tactile pulses; the shorter the time between the pulses the less download remaining. The results of their experiment showed that users were able to detect completion of a download quicker via tactile means as compared to a visual progress bar, suggesting that a tactile display could also make a successful progress indicator for mobile applications. 
The tactile progress bar used in our experiment was created to address the problems with visual progress bars by encoding progress information as a series of tactile cues which do not require visual attention and taking advantage of sensory saltation. This would give a greater perceived resolution to the progress indicator than using just a single actuator. A circular motion would be presented to the participant across three actuators (those at the lower thumb, upper thumb and the index finger), the speed of the motion indicating the rate of the download. In this case fast motion across the three actuators would indicate the download is progressing at high speed, slow motion indicating that the download is progressing at a slow speed.

The overall amount of progress was indicated by the rhythm of the vibration at each location, where a 6-note rhythm indicates that the download is in its early stages, 4-note rhythm indicates that a download is between $30 \%$ and $70 \%$ complete and a 2note rhythm indicates that the download is nearing completion. Short simultaneous vibrations at each actuator were used to indicate task completion.

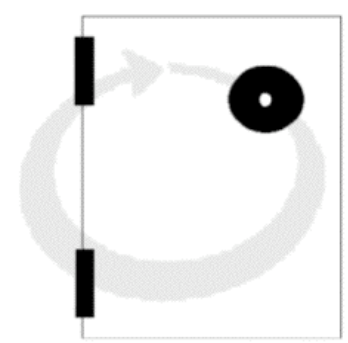

Figure 6. Illusion of clockwise circular motion for a download, where black areas represent actuators on the PDA.

By using a multi-actuator display, the tactile space is greater than in previous single-actuator studies. Only three actuators were used for displaying the download, leaving one for another task. To mimic more realistic situations where a user might be monitoring a download whilst doing some other task, alongside the tactile progress bar we included a text entry task using a typical PDA on-screen keyboard. Here the fourth actuator (on the ring finger) would provide feedback on keyboard 'slips' in the form of a long vibration of 2 secs. duration. This allowed the investigation of the effectiveness of presenting two different forms of feedback via simple vibrotactile means across four actuators. Therefore, the primary task of participants in this experiment was text entry while progress information and information on slips was presented via tactile feedback.

A 'slip' in terms of this application is defined to be a condition where the user taps down on a particular keyboard button with the stylus but, due to some external force or misguided movement, the stylus slips from that button to another before the stylus is lifted, causing either the entry of an incorrect character or no character at all (see Brewster [2] or a more detailed discussion of slips). This is a common error in mobile settings where the movement of the user and device whilst walking can cause slip errors. They are often hard to identify via the visual feedback from the buttons so tactile feedback may help users notice that an error has been made. 


\subsection{Aim and Hypotheses}

The aim of this experiment was to investigate a more realistic application for multiactuator tactile displays than Experiment 1 . This allowed us to assess the effects of tactile feedback at multiple locations on the mobile device in a more realistic scenario, and to see if the $100 \%$ recognition of locations observed in the initial experiment occurred again. The hypotheses were as follows:

1. Participants will be able to detect completion of downloads quicker with tactile feedback than a standard visual progress bar;

2. Participants will find it easier to detect slip errors when using the application with simple tactile feedback, as opposed to no tactile feedback;

3. Participants will experience reduced subjective workload due to the inclusion of tactile feedback;

4. Participants will not find masking to be a problem, as although it is likely to occur, it is unlikely to cause confusion due the large differences between the tactile feedback for progress information and the tactile slip error information.

\subsection{Experimental Design and Procedure}

Eight participants took part in the experiment, all students at the University. The experiment was a four condition within subjects design. The independent variable being the type of interface, giving four conditions to the experiment:

1. Standard visual feedback for slips and a visual progress bar;

2. Standard visual feedback for slips and tactile progress bar;

3. Tactile feedback for slips and a visual progress bar;

4. Tactile feedback for slips and tactile progress bar.

Data were recorded on the time taken to respond to the completion of a download (from when a download ended to when the participant clicked "Finished?" to indicate they had noticed its completion), the number of slip errors which remained undetected at the end of the condition, and subjective workload experienced (NASA TLX) in each condition.

The experimental tasks simulated typical mobile computing situations, where the user had to type text (in this case the text used was a poem) and monitor file downloads at the same time. The tasks involved entering as much text as possible in the time given whilst minimising errors and also monitoring the progress of the current download, detecting the completion of a download and pressing the "Finished?" button, which would initiate the next download (see Figure 7). In total, each participant was presented with five downloads per condition, where the durations were randomly selected. Each participant was allowed four minutes per interface to enter text. The participants were also permitted a five-minute training period to become comfortable with the tactile feedback at the beginning of the experiment. 


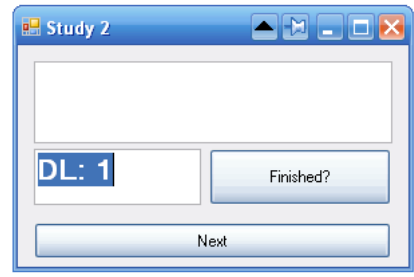

Figure 7. Display for tactile progress information.

\subsection{Results}

The results for the mean response times to download completion for each type of interface are shown in Figure 8. This being the time from completion of the download to when the user clicked finished. Where:

- Interface 1 - no feedback for slips and a visual progress bar

- Interface 2 - no feedback for slips and a tactile progress bar

- Interface 3 - tactile feedback for slips and a visual progress bar

- Interface 4 - tactile feedback for slips and tactile progress bar

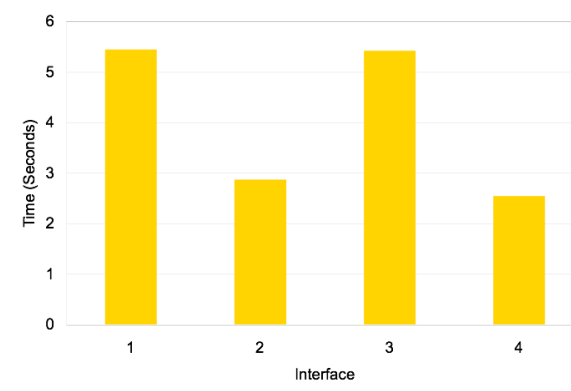

Figure 8. Mean times to respond to end of downloads.

The results indicate that the participants responded faster to the completion of downloads when using the tactile progress bar. A one-factor ANOVA was used to investigate the significance of the effect. The result shows that the differences are significant $(F=2.95)$ To identify where differences lay, Tukey's HSD tests were carried out on the data. The results of the show that there was a significant difference $(\mathrm{p}=0.05)$ between mean response times for the interfaces which used a tactile progress bar and the interfaces which used a visual progress one (with no differences within the two tactile or visual conditions).

This experiment incorporated text entry as the participants' primary task in which two of the interfaces provided tactile slip error information. The average number of slip errors undetected when using each interface is shown in Figure 9. A one-factor ANOVA showed a significant effect $(\mathrm{F}=2.94$ with $\mathrm{p}=0.05)$. 
This shows that less slip errors were left undetected by participants when using the interface with tactile slip error feedback. There was no difference in the average number of undetected errors which remained after using the interface that provided only tactile slip errors and the interface which provided both tactile progress and tactile slip error information.

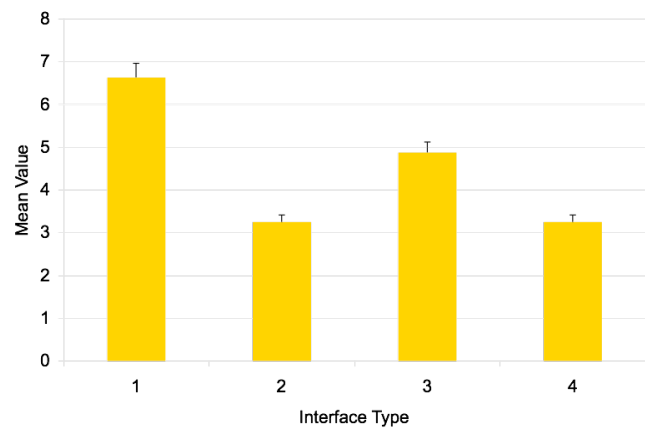

Figure 9. Average number of undetected slip errors.

As the results of the TLX workload assessments show (see Figure 10), in the case where the participant was presented with only visual slip error information the participant experienced more frustration and annoyance while the least frustration was experienced using tactile feedback for both the progress bar and slips. However any differences were not found to be statistically significant as $\mathrm{F}=2.5$ where $\mathrm{F}$ crit $=$ 3.23 .

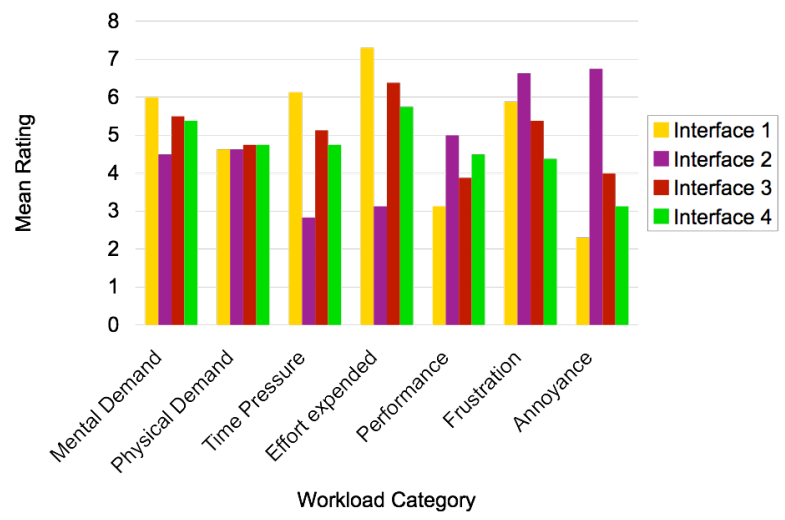

Figure 10. NASA TLX responses.

We are able to conclude that when using this particular tactile progress bar, participants were able to detect the completion of downloads faster than with the standard visual counterpart, this proves hypothesis one correct. This also confirms the result found by Brewster and King in their experiment [1].

The graph in Figure 9 shows that the participants were not only able to detect more slip errors with tactile feedback but were able to do so just as effectively when being presented this at the same time as the tactile progress information, this proves hy- 
pothesis two correct and four, partly, correct. Although we can see differences in the workload experienced by participants when using each interface, by the mean comparison in Figure 10, it was not the case that the participants experienced a reduced subjective workload by the inclusion of tactile stimuli, proving hypothesis 3 incorrect. The workload experienced was comparable to that of a standard visual based interface, this is a good basis for the introduction of tactile interfaces such as these.

The results of the statistical analysis confirm that the differences in response times to detection of completion of a download between tactile and visual interfaces were statistically significant. It also confirmed that the number of undetected slip errors showed a difference between interfaces, between the interfaces which incorporated slip information and those which did not. It was also found that less undetected errors remained after use of the interface which presented tactile progress information only. This is most likely due to the fact that the reduced effort required to monitor downloads allowed the participant to concentrate on ensuring fewer errors remained.

\section{Conclusions}

This paper presented two experiments investigating absolute identification of Tactons through a multi-actuator mobile device with a focus on potential locations on the hand to which vibrotactile information can be transferred successfully and potential applications of this technology. The results of these experiments show that an identification rate of over $87 \%$ can be achieved when two dimensions of information are encoded in Tactons using rhythm and location. They also show that location produces $100 \%$ recognition rates when using actuators situated on the mobile device at the lower thumb, upper thumb, index finger and ring finger.

The application presented in this paper is the use of Tactons presented by a mobile multi-actuator device in a tactile progress bar and in text entry error detection. Another possible application would be a waypoint navigation system. Previous work was discussed where an array of actuators was placed on a user's back [12] to provide directional information. This is not always a feasible solution as a special vest must be worn against the skin. A good alternative would be locations presented on the hand via a handheld device. This would allow a user with a PDA (or other mobile, perhaps dedicated, device) containing multiple actuators to navigate to a destination with little or no visual attention required on the mobile device.

In order to make use of multi-actuator displays in real mobile usage, future work will consider the presentation of vibrotactile information in a more realistic mobile setting, as identification may be affected by being engaged in another activity such as walking or exercising. We have refined our prototype so that it can now run from a laptop, soundcard and portable amplifier so that it now possible to use it on the move so we can investigate the effects of mobility. With further refinements we will be able to make the device self-contained.

In conclusion, this work has shown that it is possible to communicate information through four locations on the hand using multiple actuators situated on a mobile device. This is a significant step forward over single actuator displays as it now allows us to make use of the spatial dimension of tactile feedback in a convenient way. Mul- 
tiple vibrotactile actuators no longer need to be built into jackets, belts, etc. which are hard to wear; they can be placed on the actual mobile device and carried by the user. We now have at least four locations available on the hand which presents a huge tactile space for many potential applications.

\section{Acknowledgements}

This work was funded by EPSRC Advanced Research Fellowship GR/S53244. Hoggan is joint-funded by Nokia and EPSRC.

\section{References}

[1] Brewster, S. and King, A., The Design and Evaluation of a Vibrotactile Progress Bar, in Proc First Joint Eurohaptics Conference and Symposium on Haptic Interfaces for Virtual Environment and Teleoperator Systems IEEE Computer Society (2005), 499 - 500.

[2] Brewster, S. A., Overcoming the Lack of Screen Space on Mobile Computers, in Personal and Ubiquitous Computing 6(3) (2002), 188 - 205.

[3] Brewster, S. A. and Brown, L. M., Tactons: Structured Tactile Messages for Non-Visual Information Display, in Proc AUI Conference 2004, ACS (2004), 15 - 23.

[4] Brown, L. M. and Brewster, S. A., Multidimensional Tactons for Non-Visual Information Display in Mobile Devices, in Proc MobileHCI 2006, ACM Press (2006), 231 - 238.

[5] Brown, L. M., Brewster, S. A. and Purchase, H. C., A First Investigation into the Effectiveness of Tactons, in Proc WorldHaptics 2005, IEEE (2005), 167 - 176.

[6] Gaver, W., The SonicFinder: An Interface that Uses Auditory Icons in Human Computer Interaction 4(1) (1989), 67 - 94.

[7] Geldard, F. A., Sensory Saltation: Metastability in the Perceptual World, Lawrence Erlbaum Associates, New Jersey (1975)

[8] Kron, A. and Schmidt, G., Multi-Fingered Tactile Feedback from Virtual and Remote Environments in Proc 11th Symposium on Haptic Interfaces for Virtual Environment and Teleoperators, IEEE Computer Society (2003), 16.

[9] Lee, J. C., Dietz, P. H., Leigh, D., Yerazunis, W. S. and Hudson, S. E., Haptic pen: a tactile feedback stylus for touch screens in Proc 17th annual ACM symposium on User interface software and technology ACM Press (2004), 291-294.

[10] Luk, J., Pasquero, J., Little, S., MacLean, K., Levesque, V. and Hayward, V., A role for haptics in mobile interaction: initial design using a handheld tactile display prototype in Proc SIGCHI conference on Human Factors in computing systems ACM Press (2006), 171-180

[11] Myers, B. A., The Importance of Percent-Done Progress Indicators for Computer-Human Interfaces, in Proc SIGCHI conference on Human Factors in Computing Systems, ACM Press (1985), 11 - 17.

[12] Tan, H. Z. and Pentland, A., Tactual Displays for Wearable Computing, in Proc the 1st IEEE International Symposium on Wearable Computers, IEEE (1997), 84 - 89. 